\title{
Shared decision making for elective abdominal aortic aneurysm surgery
}

\author{
Authors: Michael Swart ${ }^{A}$ and Robert McCarthy ${ }^{B}$
}

\begin{abstract}
Decisions on how and when to treat an abdominal aortic aneurysm involve a number of clinicians; interventional radiologists and vascular surgeons assess the technical ability to repair the aneurysm. Patients' fitness and past medical history is assessed to estimate their short- and long-term survival with or without surgery. Most importantly the patients' personal preference for treatment must be identified. Getting a patient to share what matters most to them requires shared decision making.
\end{abstract}

KEYWORDS: Shared decision making, abdominal aortic aneurysm, perioperative medicine, open repair of abdominal aortic aneurysm, endovascular aneurysm repair

\section{Shared decision making}

Consultations with patients can be described by three different styles: check list, medical diagnosis and shared decision making. An example of a checklist consultation is the questions a patient is asked just before a medical or surgical procedure to check it is safe to proceed. The traditional medical diagnosis model of consultation uses history, examination and investigations to make a clinical diagnosis and uses a closed style of questioning. Shared decision making is used to help a patient decide to have an investigation or a procedure to diagnose or treat a medical condition. The shared decision model uses open questioning to find the patient's personal preferences. A consultation is a dynamic process and you may move between these three styles at any time.

Key features of a shared decision making consultation:

$>$ identify all the treatment options

$>$ identify the chance of benefit and harm from all the options including doing nothing

> if the chance of benefit or harm comes with uncertainty, acknowledge this uncertainty

> identify the patient's personal perspective and find out what matters to them

$>$ there are two equal experts in the consultation, the patient and the doctor

Authors: ${ }^{A}$ consultant anaesthesia and perioperative medicine, Torbay Hospital, Torquay, UK; ${ }^{B}$ consultant vascular surgeon, Torbay Hospital, Torquay, UK
> reach a decision and agree a plan of action or repeat the whole process at a later date.

The term 'shared decision making' first appeared in medical ethics publications in the 1970s. It came to prominence in 1982 when it was used to extend the concept of informed consent in the US president's Commission for the Study of Ethical Problems in Medicine and Biomedical and Behavioral Research. ${ }^{1}$ In 2011 , Coulter and Collins published Making shared decision making a reality; this document and publications by Glyn Elwyn are good starting points to learn more about the clinical use of shared decision making. ${ }^{2-4}$ The medico-legal imperative to use shared decision has been increased by the Montgomery judgement. ${ }^{5}$ This is explored in more detail by Sturgess. ${ }^{6}$ Spread and adoption of shared decision making has been slow. A recent systematic review on shared decision making and surgery found that surgeons perceived their consultations as shared decision making more than their patients ( $44 \%$ vs $29 \%$, respectively). ${ }^{7}$

\section{Abdominal aortic aneurysms}

Men aged 65 years have approximately a $1 \%$ chance of having abdominal aortic aneurysms (AAA), defined as an aortic diameter $>3 \mathrm{~cm}$, this prevalence increases with age and men are four to six times more likely to have an AAA than women. Men aged 65 years in the UK are offered AAA screening by ultrasound and there are similar screening programmes in most developed world healthcare systems. Men with aneurysms are followed up with further ultrasound scans. If the aneurysm reaches $5.5 \mathrm{~cm}$, they are referred to a vascular surgeon. Aneurysms may be diagnosed as an incidental finding from computed tomography (CT), magnetic resonance imaging (MRI) or ultrasound, and some are found by abdominal palpation. It is uncommon for an AAA to cause any symptoms until the event of rupture so most patients are unaware of the aneurysm until the point that it is diagnosed.

At $5.5 \mathrm{~cm}$, CT angiography is performed to further define the anatomy of the aneurysm and the patient is assessed for fitness for surgery. Data from four prospective randomised controlled studies demonstrates that below $5.5 \mathrm{~cm}$, the chance of death after surgery is greater than the chance of death from the aneurysm rupturing. ${ }^{8}$ These studies show that open repair of an AAA less than $5.5 \mathrm{~cm}$ increased short-term mortality by 10 times without any long-term benefit.

There is poor data studying the effects of surgery on AAAs greater than $5.5 \mathrm{~cm}$. There is one prospective randomised controlled study on AAA greater than $5.5 \mathrm{~cm}$. This is the EVAR 
Table 1. Risk of rupture per year as abdominal aortic aneurysm increases in size

\begin{tabular}{|c|c|c|c|}
\hline $\begin{array}{l}\text { AAA } \\
\text { diameter }\end{array}$ & Description & $\begin{array}{l}\text { Chance of } \\
\text { rupture in } \\
\text { next year }\end{array}$ & $\begin{array}{l}\text { Chance of } \\
\text { no rupture }\end{array}$ \\
\hline$<3.9 \mathrm{~cm}$ & $\begin{array}{l}\text { Very small } \\
\text { aneurysm }\end{array}$ & No real risk & Very high \\
\hline $4.0-4.9 \mathrm{~cm}$ & Small aneurysm & About 1 in 100 & $\begin{array}{l}\text { About } 99 \text { in } \\
100\end{array}$ \\
\hline $5.0-5.9 \mathrm{~cm}$ & Aneurysm & About 1 in 12 & About 11 in 12 \\
\hline $6.0-6.9 \mathrm{~cm}$ & Large aneurysm & About 1 in 6 & About 5 in 6 \\
\hline$>7.0 \mathrm{~cm}$ & $\begin{array}{l}\text { Very large } \\
\text { aneurysm }\end{array}$ & $\begin{array}{l}\text { About } 1 \text { in } 4 \\
\text { or higher }\end{array}$ & About 3 in 4 \\
\hline
\end{tabular}

2 study where patients with an $A A A>5.5 \mathrm{~cm}$ but deemed too unfit for open surgery were randomised to either best medical treatment or endovascular aneurysm repair (EVAR): this showed no survival benefit from EVAR. ${ }^{9}$

The risk of an AAA rupture is primarily related to AAA diameter; if the aneurysm increases in size the chance of rupture goes up (Table 1). ${ }^{10}$

There are three treatment options for an AAA.

> Best medical treatment with either no follow-up or continued surveillance. This includes treating hypertension, high cholesterol, smoking cessation and exercise.

$>$ Open aneurysm repair.

> EVAR where a stent is inserted within the aorta to exclude the aneurysm. The option of EVAR depends on adequate access and the anatomical morphology of the aorta.

The advantages of open repair are that it does not require followup and has less chance of requiring further secondary surgical intervention; the disadvantage of an open surgery is a higher short-term mortality and morbidity with a longer recovery period. The advantage of an EVAR is a lower short-term mortality and morbidity with a quicker recovery and the disadvantage of an EVAR is continued radiological follow-up, a higher chance of additional surgical intervention and possibly a shorter long-term survival. ${ }^{11,12}$

\section{Perioperative medicine clinic}

The first description of a perioperative medicine clinic was published in $1949 .^{13}$ This clinic described by J Alfred Lee is similar in structure to today's clinics, however the investigations now used to assess patients are different. The surgical population and volume of cases treated has changed. We are doing more surgery on an older population with more comorbidities. ${ }^{14}$ Over the last 15 years, perioperative medicine clinics run by anaesthetists and proactive care of older people (POPS) clinics run by geriatricians have increased in number to deal with the medical complexities of the surgical patient. ${ }^{15}$

These clinics provide the opportunity to optimise medical conditions such as anaemia, atrial fibrillation and diabetes or to liaise with other medical specialties. There is also the opportunity to refer patients to smoking cessation and exercise or weight loss programmes and to dieticians for either weight loss or weight gain.
In addition, the postoperative plan for high dependency care or intensive care can be determined. Other aspects of postoperative care such as management of comorbidities and drug therapy can also be planned.

The main purpose in operating on an AAA is to prevent AAA rupture and increase the patient's long-term survival. The traditional outcome measure for AAA surgery is 30 -day survival. Both 30-day survival and median survival with or without surgery can be estimated for an individual patient; we use a validated prediction model for survival after AAA repair that uses preoperative variables. ${ }^{16}$ The survival prediction model uses age, sex, weight, height, haemoglobin, creatinine, peak oxygen consumption, ventilatory equivalents for carbon dioxide at the anaerobic threshold, and a past medical history of peripheral arterial disease, heart failure, stoke or transient ischaemic attack and myocardial infarction or angina. The peak oxygen consumption and ventilatory equivalents for carbon dioxide are measured by a cardiopulmonary exercise test (CPET), if this is not available they can be estimated; CPET is available in over $50 \%$ of UK hospitals that perform elective surgery. ${ }^{17}$

The most important part of the perioperative medicine clinic for patients with an AAA is the shared decision-making consultation. We have a 1-hour appointment for the CPET and the consultation. The CPET provides information to asses the impact of disease on the patient's cardiorespiratory reserves when put under the stress of an exercise bike, it also allows time to build a relationship with the patient to facilitate the shared decision-making process. The standard vascular surgical clinic appointment time is 15 minutes, which may be long enough to explain the surgical options but is not long enough to explore the patient's personal values in detail.

\section{Vascular multidisciplinary team meeting}

All patients with an AAA have their treatment options discussed at a vascular multidisciplinary team (MDT) meeting. At the meeting, perioperative medicine, interventional radiologists, vascular specialist nurses and vascular surgeons review the patient's options. The CT angiography is reviewed to determine if EVAR is simple, difficult or complex and whether referral for a tertiary service review is appropriate or not. The advantages, disadvantages and complexity of open AAA repair are reviewed. The patient's predicted short- and long-term survival is discussed and, most importantly, the patient's personal perspective is reviewed

At the end of the discussion, a plan to communicate to the patient their treatment options is made. This is usually done by a vascular surgeon in the vascular surgery clinic.

\section{Case studies}

The following three case studies explore some of the aspects of shared decision making for AAA surgery. None of the cases are real patients. They are fictional but use our experience over the last 15 years for examples of shared decision making for patients considering high-risk major surgery.

\section{Case 1}

Mr A is 60-year-old builder. He had CT to investigate lower back pain. An incidental finding on this $C T$ was a $5.7 \mathrm{~cm}$ AAA. His past medical history included drug therapy to treat hypertension and 


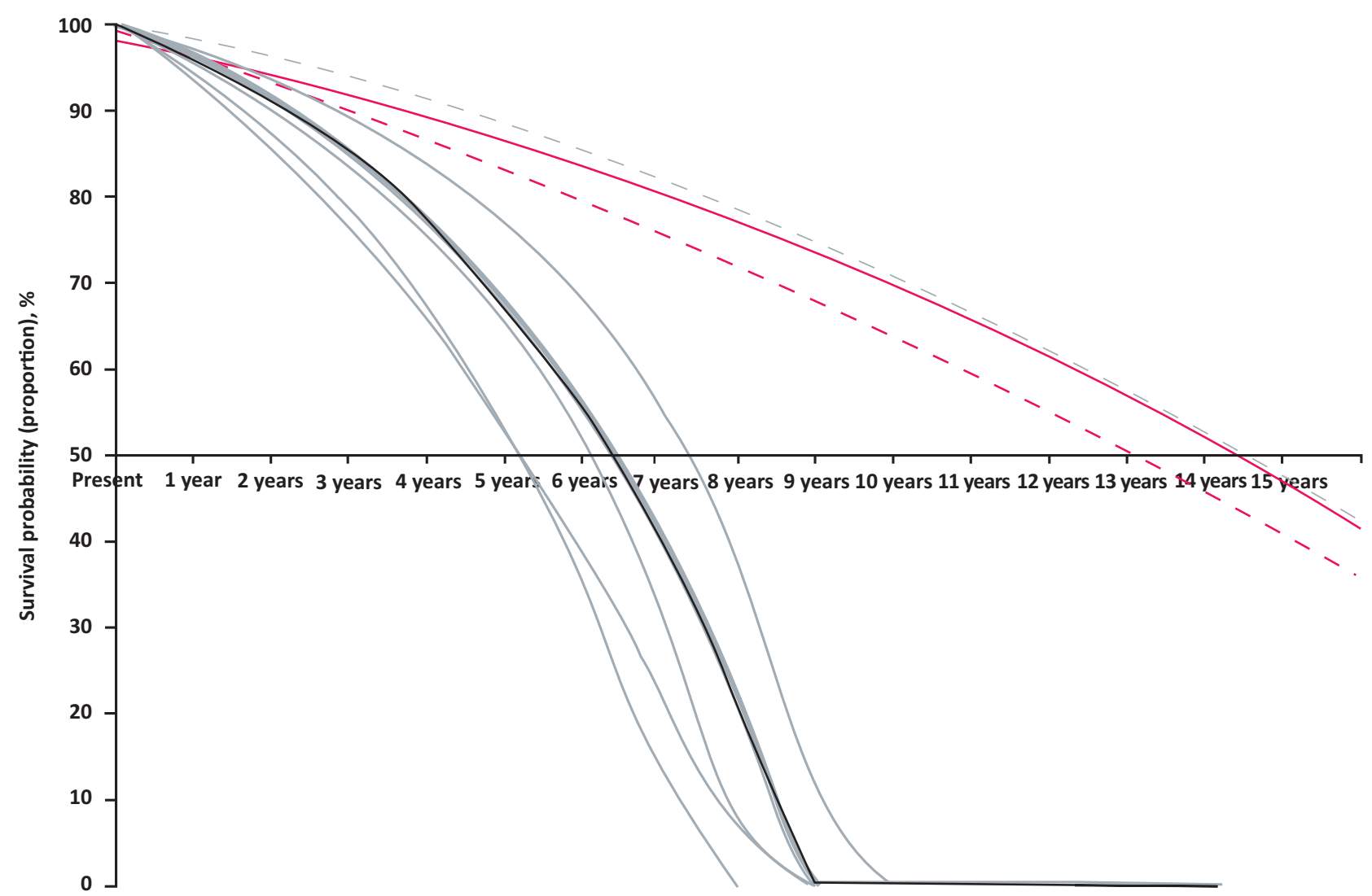

Fig 1. Predicted survival curves for a patient ( $\mathrm{Mr} \mathrm{A}$ ) with abdominal aortic aneurysm without surgery (solid black line), after open repair (solid red line) and after endovascular repair (red dashes), and predicted survival with no abdominal aortic aneurysm (grey dashes). The rate of aneurysm expansion is uncertain, illustrated by four grey lines, generated with four other equations.

high cholesterol. His predicted 30-day mortality for an EVAR is $0.5 \%$ and the equivalent for an open AAA repair is $2 \%$. Median predicted survival with no surgery is 6.5 years, with EVAR it is 13 years and with open surgery 14 years (Fig 1). The view of the vascular MDT meeting was that both EVAR and open surgery were options, but his best option was open surgery. This was because his open 30-day mortality was low and his long-term survival may out last the EVAR and he could require a secondary surgical intervention AAA in the future. When informed about all the chances of benefit and harm Mr A chose EVAR to treat his AAA because he wanted as short a recovery as possible so that he could return to work as a self-employed builder.

\section{Case 2}

$\mathrm{Mr}$ B is a 75-year-old man. He had a $5.5 \mathrm{~cm}$ screen detected AAA. He had renal impairment, peripheral arterial disease and has had a myocardial infarction in the past. His predicted 30-day mortality was $10 \%$ for open AAA surgery and $3 \%$ for EVAR. His median survival was 4.5 years with or without surgery (Fig 2). The vascular MDT meetings opinion was that there was no survival benefit from surgery. EVAR was technically an option. Open surgery was possible but the chance of postoperative mortality or life changing morbidity was too high to justify with no survival benefit. The plan was to communicate with him the no survival benefit and the option for EVAR only if there was a personal preference to justify taking the chance of postoperative mortality and morbidity. Mr B has a son in America. He wanted to visit his son before he dies. Now that he had a $5.5 \mathrm{~cm}$ AAA, he could no longer get travel insurance to go to America. He opted for an EVAR. He survived the surgery but postoperative complications had a long-term impact on his mobility and he was too unfit to travel to America.

\section{Case 3}

$\mathrm{MrC}$ is an 82-year-old man and had a $6.5 \mathrm{~cm}$ AAA diagnosed after $\mathrm{CT}$ to investigate abdominal pain. He had no other significant past medical history. He had an excellent CPET and was of above average fitness for his age. Predicted 30-day mortality for open AAA repair was $6 \%$ and for EVAR was $2 \%$. Median survival without surgery was 5 years and with surgery was 7 years (Fig 3 ). The consensus view of the vascular MDT is that EVAR was not an option because of the anatomy of the aneurysm. There was a survival benefit from open surgery and this was thought to be his best option. $\mathrm{Mr} \mathrm{C}$ declined surgery. His wife had severe dementia and lived in a care home; he had no other friends or family. He was her only visitor and he could not take any chance of a postoperative complication that would prevent him from visiting his wife.

\section{Discussion}

Assessment of surgical intervention to treat an AAA and prediction of survival with or without surgery require knowledge and experience. These skills are repetitive tasks that are relatively easy 


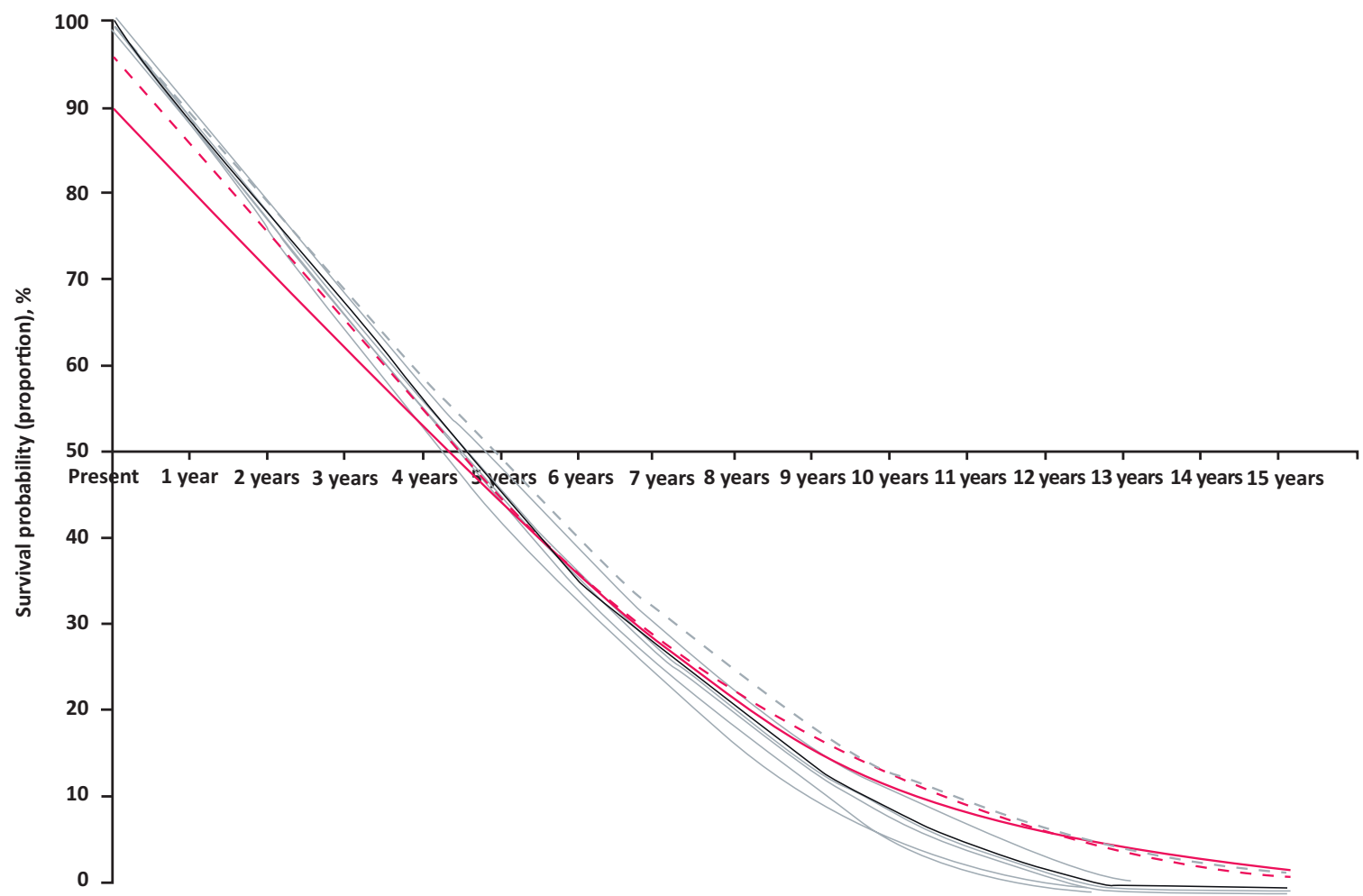

Fig 2. Predicted survival curves for a patient ( $\mathrm{Mr}$ B) with abdominal aortic aneurysm without surgery (solid black line), after open repair (solid red line) and after endovascular repair (red dashes), and predicted survival with no abdominal aortic aneurysm (grey dashes). The rate of aneurysm expansion is uncertain, illustrated by four grey lines, generated with four other equations.

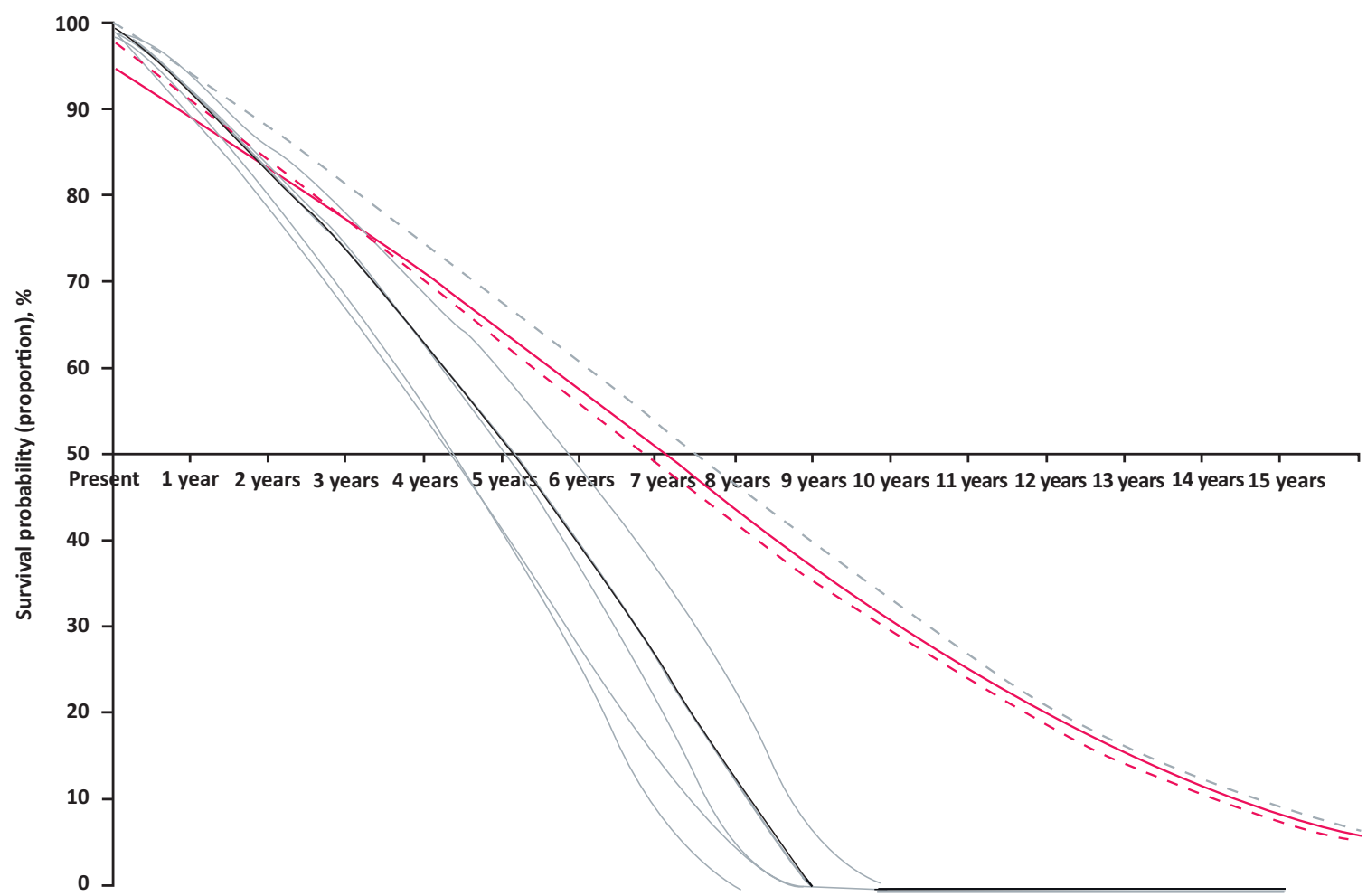

Fig 3. Predicted survival curves for a patient ( $\mathrm{MrC}$ ) with abdominal aortic aneurysm without surgery (solid black line), after open repair (solid red line) and after endovascular repair (red dashes), and predicted survival with no abdominal aortic aneurysm (grey dashes). The rate of aneurysm expansion is uncertain, illustrated by four grey lines, generated with four other equations. 
to teach and learn. Patients' personal preferences come in many forms and are often unpredictable from the clinicians' perspective. Indeed, some clinicians, especially if using the traditional medical model of consultation, may not explore such issues or fail to address patient specific concerns. What the patient wants can only be found by giving the patient the appropriate environment to feel comfortable to open up and share their thoughts with you. Shared decision making is a more difficult skill to teach or learn.

When in training, doctors want to acquire knowledge and skills that focus on diagnosis, treatment and technical skills to perform procedures; this is also the focus of postgraduate examinations and specialist accreditation. In a consultation when a doctor comes across uncertainty, they tend to resort to discussing their knowledge on something they feel more certain and comfortable. This leads them to discussing the evidence of one technique over another. This evidence comes through medical research where the outcomes are determined by doctors and not patients. It shifts the consultation towards medical decision making and away from shared decision making.

A key part of the decision making comes from communication within the vascular MDT. The MDT meeting is a formal documentation of discussion and treatment plans. Inside the meeting there must be the appropriate environment for all opinions to be shared openly. There is also a great deal of both formal and informal communication outside the meeting. The MDT meeting needs to be actively and fairly chaired to allow all information to be weighed up before treatment options are shared with the patient. It is vital that MDT discussions and decisions are recorded accurately for future learning for all the clinicians involved in the MDT.

Most of the MDT outcomes are communicated to the patient via the vascular surgeon. This is because they are usually the first point of contact with the patient and interventional radiologists traditionally do not have clinics. For complex patients, a bespoke joint surgical, radiological and perioperative medicine consultation is arranged.

Other aspects that are raised in the perioperative medicine clinic are discussions on end-of-life care if postoperative complications occur. For patients who decide not to have surgery for their AAA a 'what happens if it ruptures' discussion takes place. The patient, and often their partner, are usually thinking about this but find it difficult to start a discussion on the matter.

Shared decision making is at the centre of all good medicine, it takes time to learn, develop and feel comfortable to use these skills. We strongly believe that adopting such an open, inclusive and patient-centric focus will improve patient decision making and less decisional regret by both patients and clinicians. The rewards for the patient and the clinician make this a worthwhile investment.

\section{Acknowledgements}

We would like to thank Dr John Carlisle for producing the figures.

\section{References}

1 President's Commission for the Study of Ethical Problems in Medicine and Biomedical and Behavioral Research. Making health care decisions: The ethical and legal implications of informed consent in the patient-practitioner relationship. Washington: US Government Printing Office, 1982:33.

2 Coulter A, Collins A. Making shared decision-making a reality. No decision about me without me. London: The Kings Fund, 2011.

3 Elwyn G, Frosch D, Thomson R et al. Shared decision making: a model for clinical practice. J Gen Intern Med 2012;27:1361-7.

4 Elwyn G, Durand MA, Song ] et al. A three-talk model for shared decision making: multistage consultation process. BMJ 2017;359:j4891.

5 The Supreme Court. Montgomery (Appellant) v Lanarkshire Health Board (Respondent) (Scotland): [2015]UKSC 11: On appeal from: [2013]CSIH 3. The Supreme Court, 2015.

6 Sturgess J, Clapp JT, Fleisher LA. Shared decision making in perioperative medicine: a narrative review. Anaesthesia 2019;74(Suppl 1): 13-9.

7 De Mik SML, Stubenrouch FE, Balm R et al. Systematic review of shared decision making in surgery. BJS 2018;105:1721-30.

8 Powell JT, Sweeting MJ, Ulug P et al. Meta-analysis of individualpatient data from EVAR-1, DREAM, OVER and ACE trials comparing outcomes of endovascular or open repair for abdominal aortic aneurysm over 5 years. Br J Surg 2017;104:166-78.

9 United Kingdom EVAR Trial Investigators, Greenhalgh RM, Brown LC et al. Endovascular repair of aortic aneurysm in patients physically ineligible for open repair. N Eng J Med 2010;362:1872-80.

10 Brewster DC, Cronenwett JL, Hallett JW Jr et al. Guidelines for the treatment of abdominal aortic aneurysms. Report of a subcommittee of the Joint Council of the American Association for Vascular Surgery and Society for Vascular Surgery. J Vasc Surg 2003;37:1106-17.

11 Schermerhorn ML, Buck DB, O'Maaey AJ et al. Long-term outcome of abdominal aortic aneurysm in the Medicare population. N Eng J Med 2015;373:328-38.

12 Lederle FA, Kriakides TC, Stroupe KT et al. Open versus endovascular repair of abdominal aortic aneurysm. N Eng J Med 2019;380:2126-35.

13 Lee JA. An anaesthetic out-patient clinic. Anaesthesia 1949;4:169-74.

14 Barnett K, Mercer SW, Norbury M et al. Epidemiology of multimorbidity and implications for health care, research and medical education: a cross-sectional study. Lancet 2012;380:37-43.

15 Dhesi JK, Swart M. Specialist pre-operative assessment clinics. Anaesthesia 2016;71(Suppl 1):3-8.

16 Carlisle JB, Danjoux G, Kerr K, Snowden C, Swart M. Validation of long term survival prediction for scheduled abdominal aortic aneurysm repair with independent calculator using only preoperative variables. Anaesthesia 2015;70:654-65.

17 Reeves T, Bates S, Sharp T et al Cardiopulmonary exercise testing (CPET) in the United Kingdom - national survey of the structure, conduct, interpretation and funding. Perioperative Medicine 2018;7:2.

Address for correspondence: Dr Michael Swart, Torbay Hospital, Torquay, Devon TQ2 7AA, UK Email: michael.swart@nhs.net 